\title{
Simulating Properties of the Likelihood Ratio Test for a Unit Root in an Explosive Second Order Autoregression
}

\author{
Bent Nielsen \\ Nuffield College, University of Oxford \\ J. James Reade \\ St. Cross College, University of Oxford
}

October 19, 2004

\begin{abstract}
This paper provides a means of accurately simulating explosive autoregressive processes, and uses this method to analyse the distribution of the likelihood ratio test statistic for an explosive second order autoregressive process. Nielsen (2001) has shown that for the asymptotic distribution of the likelihood ratio unit root test statistic in a higher order autoregressive model, the assumption that the remaining roots are stationary is unnecessary, and as such the approximating asymptotic distribution for the test in the difference stationary region is valid in the explosive region also. However, simulations of statistics in the explosive region are beset by the magnitude of the numbers involved, which cause numerical inaccuracies, and this has previously constituted a bar on supporting asymptotic results by means of simulation, and analysing the finite sample properties of tests in the explosive region.
\end{abstract}

\section{Introduction}

This paper provides a means of accurately simulating explosive autoregressive processes, and uses this method to analyse the distribution of the likelihood ratio test statistic for an explosive second order autoregressive process. Nielsen (2001) has shown that for the asymptotic distribution of the likelihood ratio unit root test statistic in a $p^{\text {th }}$ order autoregressive model, $p>1$, the assumption that the remaining roots are stationary is unnecessary, and as such the approximating asymptotic distribution for the test in the difference stationary region is valid in the explosive region also. However, simulations of statistics in the explosive region are beset by the magnitude of the numbers involved, which cause numerical inaccuracies. Often the use of logarithms can alleviate such problems; however with the likelihood ratio statistic there is no simple way to incorporate logarithms to such an effect. 
This has previously constituted a bar on supporting asymptotic results by means of simulation, and analysing the finite sample properties of tests in the explosive region.

Anderson (1959) has provided a number of convergence results for the Ordinary Least Squares estimator in an explosive first order autoregressive process, allowing numerically stable simulations to be carried out throughout the parameter space. It will be shown how these results can be applied to the second order autoregressive model to allow the likelihood ratio test statistic to be accurately simulated in the explosive case. This is then used to address two issues: first that the asymptotic analysis of the test statistic can be supported by simulation and second that the finite sample distribution of the test statistic can be approximated accurately by a local asymptotic approach also in the explosive case.

\section{The Simulation Problem}

In the following the underlying statistical model of the likelihood ratio test statistic for the unit root hypothesis is presented, and then follows the asymptotic theory and a description of the simulation issue.

\subsection{The Statistical Model and the Null Hypothesis}

Suppose a time series $X_{-1}, X_{0}, X_{1}, \ldots, X_{T}$ is observed and consider the univariate second order autoregressive model given by:

$$
X_{t}=\alpha_{1} X_{t-1}+\alpha_{2} X_{t-2}+\varepsilon_{t},
$$

where the initial values, $X_{0}$ and $\Delta X_{0}$ are fixed and the innovations $\varepsilon_{t}$ are independently identically $N\left(0, \sigma^{2}\right)$ distributed and the parameters $\alpha_{1}, \alpha_{2}$ and $\sigma$ vary freely. The model can be transformed into

$$
\Delta X_{t}=\Pi X_{t-1}+\zeta \Delta X_{t-1}+\varepsilon_{t},
$$

where $\Pi=\alpha_{1}-1+\alpha_{2}$ and $\zeta=-\alpha_{2}$. The unit root test hypothesis is:

$$
\mathrm{H}_{0}: \Pi=0 .
$$

For the finite sample analysis of Section 4.3 it is convenient to reparameterise this as:

$$
\Delta^{2} X_{t}=\Pi X_{t-1}-\Gamma \Delta X_{t-1}+\varepsilon_{t},
$$

where $\Gamma=1+\alpha_{2}=1-\zeta$. When $\Pi=0$ the parameter $\Gamma$ determines the stochastic properties of the process: when $0 \leq \Gamma \leq 2$ the process is difference stationary, when $\Gamma=0$ it is $\mathrm{I}(2)$, and when $|1-\Gamma|>1$ it is explosive. 


\subsection{The Test Statistic}

The model is analysed by regressing $\Delta X_{t}$ and $X_{t-1}$ on $\Delta X_{t-1}$ giving the residuals:

$$
\begin{aligned}
& R_{0, t}=\left(\Delta X_{t} \mid \Delta X_{t-1}\right)=\Delta X_{t}-\frac{\sum_{t=1}^{T} \Delta X_{t} \Delta X_{t-1}}{\sum_{t=1}^{T} \Delta X_{t-1}^{2}} \Delta X_{t-1}, \\
& R_{1, t}=\left(X_{t-1} \mid \Delta X_{t-1}\right)=X_{t-1}-\frac{\sum_{t=1}^{T} X_{t-1} \Delta X_{t-1}}{\sum_{t=1}^{T} \Delta X_{t-1}^{2}} \Delta X_{t-1} .
\end{aligned}
$$

Defining the partial correlation of $\Delta X_{t}$ and $X_{t}$ given by $S_{i j}=T^{-1} \sum_{t=1}^{T} R_{i, t} R_{j, t}^{\prime}$ and the sample product moments $\lambda^{2}=S_{01}^{2} / S_{00} S_{11}$, the likelihood ratio test statistic can be written as:

$$
\mathrm{LR}=-T \log \left(1-\lambda^{2}\right)
$$

\subsection{The Asymptotic Theory}

The asymptotic result which is hoped to be checked by simulation stems from Nielsen (2001). This shows that the standard Dickey-Fuller distribution can be used for unit root testing for all values of $\Gamma$ in the parameterisation, except when $\Gamma=0$ as the process is $\mathrm{I}(2)$.

Theorem 2.1 In the second order autoregressive model in (3), the null distribution of the likelihood ratio test statistic for $\mathrm{H}_{0}$ can be approximated as follows for $T \rightarrow \infty$ and fixed value of $\Gamma$ and $\sigma^{2}$. Let $W$ be a univariate standard Brownian motion, then:

$$
\mathrm{LR} \stackrel{d}{\longrightarrow}\left(\int_{0}^{1} W_{u} d W_{u}\right)^{2} / \int_{0}^{1} W_{u}^{2} d u \quad \text { for } \Gamma \neq 0
$$

The finite sample properties of the test are distorted by a non-continuity at $\Gamma=0$. To bridge over the difficult asymptotic distribution Nielsen (2004) formulated the following local asymptotic result

Theorem 2.2 In the second order autoregressive model in (3), the null distribution of the likelihood ratio test statistic for $\mathrm{H}_{0}$ can be approximated as follows for $T \rightarrow \infty$ and fixed value of $\gamma=\Gamma T$ and $\sigma^{2}$. Let $V$ be the univariate Ornstein-Uhlenbeck process $V_{u}$ given by $V_{v}=-\gamma \int_{0}^{v} V_{u} d u+W_{v}$ for a standard Brownian motion, $W$, and let $\widetilde{V}_{v}=\int_{0}^{v} V_{u} d u$ and $\widehat{V}_{v}=\widetilde{V}_{v}-V_{v} \int_{0}^{1} \widetilde{V}_{u} V_{u} d u / \int_{0}^{1} V_{u}^{2} d u$. Then:

$$
\mathrm{LR} \stackrel{d}{\longrightarrow}\left(\int_{0}^{1} \widehat{V}_{u} d V_{u}\right)^{2} / \int_{0}^{1} \widehat{V}_{u}^{2} d u .
$$

In a simulation analysis of the difference stationary situation, $0<\Gamma<2$, Nielsen (2004) found that the local asymptotic approximation works rather well in finite samples. A second aim of this paper is to explore this in the explosive case. 
Table 1: Simulated expected value of likelihood ratio test statistic for range of explosive and non-explosive parameterisations. Based on $10^{6}$ replications. The asymptotic figures are taken from Nielsen (1997; 2004).

\begin{tabular}{lccccccc}
\hline \multicolumn{7}{c}{$\mathrm{T}$} \\
$\Gamma$ & 6 & 12 & 24 & 48 & 96 & 192 & $\infty$ \\
\hline$-8 / 3$ & 1.638 & 1.358 & 1.244 & $\mathbf{5 5 . 1 9 0}$ & $\mathbf{1 0 9 . 9 2 0}$ & $\mathbf{N a N}$ & 1.142 \\
$-4 / 3$ & 1.829 & 1.459 & 1.297 & $\mathbf{6 . 5 9 8}$ & $\mathbf{6 3 . 1 7 3}$ & $\mathbf{1 2 6 . 3 3 0}$ & $\vdots$ \\
$-2 / 3$ & 1.686 & 1.671 & 1.402 & 1.271 & $\mathbf{3 9 . 4 5 4}$ & $\mathbf{7 9 . 2 4 7}$ & $\vdots$ \\
$-1 / 3$ & 1.656 & 1.592 & 1.615 & 1.378 & 1.259 & $\mathbf{5 9 . 1 5 8}$ & $\vdots$ \\
$-1 / 6$ & 1.689 & 1.445 & 1.618 & 1.594 & 1.368 & 1.254 & $\vdots$ \\
$-1 / 12$ & 1.707 & 1.463 & 1.400 & 1.645 & 1.584 & 1.363 & $\vdots$ \\
$-1 / 24$ & 1.715 & 1.479 & 1.411 & 1.382 & 1.662 & 1.582 & 1.142 \\
0 & 1.722 & 1.491 & 1.437 & 1.412 & 1.405 & 1.404 & 1.402 \\
$1 / 24$ & 1.727 & 1.495 & 1.435 & 1.385 & 1.321 & 1.255 & 1.142 \\
$1 / 12$ & 1.730 & 1.490 & 1.409 & 1.327 & 1.255 & 1.207 & $\vdots$ \\
$1 / 6$ & 1.730 & 1.464 & 1.344 & 1.257 & 1.205 & 1.177 & $\vdots$ \\
$1 / 3$ & 1.706 & 1.390 & 1.267 & 1.207 & 1.175 & 1.160 & $\vdots$ \\
$2 / 3$ & 1.607 & 1.293 & 1.212 & 1.175 & 1.158 & 1.150 & $\vdots$ \\
$4 / 3$ & 1.439 & 1.226 & 1.179 & 1.158 & 1.148 & 1.145 & $\vdots$ \\
$8 / 3$ & 1.269 & 1.150 & 1.136 & 1.135 & $\mathbf{6 0 . 7 0 8}$ & $\mathbf{1 2 2 . 3 8 0}$ & 1.142 \\
\hline
\end{tabular}

\section{$2.4 \quad$ Initial Simulation Results}

In Table 1 the expected value of the LR test statistic is reported from a Monte Carlo simulation of one million repetitions. The simulations are done in the usual way: for each repetition a time series $X_{1}, \ldots, X_{T}$ with initial values $X_{0}=\Delta X_{0}=0$ is simulated and the likelihood ratio test statistic from (6) is then computed using double precision. One million repetitions provides a standard error of about $1 \times 10^{-3}$, allowing confidence in results to about the third decimal place. In Table 1 the results for the range of values $0 \leq \Gamma \leq 4 / 3$ replicate the work of Nielsen (2004), and appear to be based on numerically stable computations. In contrast, the highlighted values outside this region indicate some numerical problems, with one entry uncomputable despite the double precision and thus indicated by NaN for 'Not a Number'. We will propose a solution for numerically stable simulations that will give a version of Table 1 that can be used for assessing the suitability of the asymptotic results in Theorems 2.1 and 2.2 . 
In a related paper Basawa, Mallik, McCormick and Taylor (1989) prove the asymptotic validity of the bootstrap for the least squares estimate in an explosive first order autoregression. They support their analysis with a simulation for a single parameter value of $\beta=1.05$ with $T=200$. This choice corresponds to the entry $\Gamma=1 / 24$ and $T=192$ in Table 1 where standard simulations appear numerically stable.

\section{The Proposed Solution}

Anderson (1959) gave the asymptotic theory for the ordinary least squares estimator in an explosive first order autoregressive process, and the solution to the numerical instability problem can be found in the proof of these results.

In order to utilise Anderson's technique some algebraic manipulations of the $S_{i j}$ expressions are needed. Imposing the null hypothesis in (2) gives:

$$
\Delta X_{t}=\zeta \Delta X_{t-1}+\varepsilon_{t}
$$

In the explosive case, $|\zeta|>1$, the differences process $\Delta X_{t}$ satisfies an explosive first order autoregressive as in Anderson's setup. Based on (8) the residuals $R_{0, t}$ reduce to:

$$
R_{0, t}=\left(\Delta X_{t} \mid \Delta X_{t-1}\right)=\left(\varepsilon_{t} \mid \Delta X_{t-1}\right) .
$$

Next, subtracting $\zeta \Delta X_{t}$ from both sides of (8) and taking sums:

$$
(1-\zeta) X_{t-1}=-\zeta \Delta X_{t-1}+\sum_{s=0}^{t-1} \varepsilon_{s}
$$

Using this expression the residuals $R_{1, t}$ then satisfy, for $\zeta \neq 1$ :

$$
(1-\zeta) R_{1, t}=(1-\zeta)\left(X_{t-1} \mid \Delta X_{t-1}\right)=\left(\sum_{s=0}^{t-1} \varepsilon_{s} \mid \Delta X_{t-1}\right) .
$$

Then (9) and (11) can be used to rewrite, for instance, $S_{01}$ as follows:

$$
\begin{aligned}
(1-\zeta) S_{01}=\frac{1}{T} \sum_{t=1}^{T} \varepsilon_{t} & \left(\sum_{s=1}^{t} \varepsilon_{s}\right) \\
& -\frac{\left[\frac{1}{T} \sum_{t=1}^{T}\left(\sum_{s=1}^{t} \varepsilon_{s}\right) \Delta X_{t-1}\right]\left(\frac{1}{T} \sum_{t=1}^{T} \varepsilon_{t} \Delta X_{t-1}\right)}{\frac{1}{T} \sum_{t=1}^{T} \Delta X_{t-1}^{2}},
\end{aligned}
$$

Equation (12) is the alternative expression for the $S_{01}$ term which can be used to approximate the likelihood ratio test statistic. Analogous transformations of $S_{00}$ and $S_{11}$ can be found using (9) and (11) respectively. They all have the property that the first term in each expression converges to a non-explosive quantity while 
the second term vanishes, that is $S_{i j}=O_{p}(1)-o_{p}(1)$. It remains however, that in finite samples the second term in each equation cannot be simulated accurately by the usual technique due to numerical instability; instead a method of simulating them using the proof of Anderson will be outlined.

Anderson defined the processes $z_{t}$ and $y_{t}$ :

$$
\begin{aligned}
& z_{t}=\beta^{t-2} \Delta X_{t-1}=\alpha \Delta X_{0}+\varepsilon_{1}+\beta \varepsilon_{2}+\ldots+\beta^{t-2} \varepsilon_{t-1}, \\
& y_{t}=\varepsilon_{t}+\beta \varepsilon_{t-1}+\ldots+\beta^{t-2} \varepsilon_{2}+\beta^{t-1} \varepsilon_{1}=\sum_{s=1}^{t} \beta^{t-s} \varepsilon_{s},
\end{aligned}
$$

where $\beta=1 / \zeta$. Equation (13) is a non-explosive representation of the differenced autoregressive process, while (14) is a moving average representation. Using the proofs of Anderson's Theorems 2.1 and 2.2, it can be written that:

$$
\begin{aligned}
& \beta^{2(T-2)} \sum_{t=1}^{T} \Delta X_{t-1}^{2}=\frac{1}{1-\beta^{2}} z_{T}^{2}+\sum_{s=1}^{T-1} \beta^{2 s}\left(z_{T-s}^{2}-z_{T}^{2}\right)-\frac{\beta^{2 T}}{1-\beta^{2}} z_{T}^{2}, \\
& \beta^{T-2} \sum_{t=1}^{T} \varepsilon_{t} \Delta X_{t-1}=y_{T} z_{T}+\sum_{t=1}^{T-1} \beta^{t} \varepsilon_{T-t}\left(z_{T-t}-z_{T}\right) .
\end{aligned}
$$

In the asymptotic analysis the idea is to show that the second and third terms on the right hand side of (15) and (16) converge to zero as $T \rightarrow \infty$. Thus asymptotically $\beta^{2(T-2)} \sum_{t=1}^{T} \Delta X_{t-1}^{2}$ can be well approximated by $z_{T}^{2} /\left(1-\beta^{2}\right)$, which is convergent due to the Marcinkiewicz-Zygmund result (see Lai and Wei, 1983), and $y_{T} z_{T}$ can be used to approximate $\beta^{T-2} \sum_{t=1}^{T} \varepsilon_{t} \Delta X_{t-1}$. This allows accurate approximation of $S_{00}$, and this is done by first generating the time series $y_{t}$ and $z_{t}$ for each replication and then simulating the expressions on the right hand side of equations (15) and (16).

For $S_{01}$ and $S_{11}$ to be simulated, the integrated error term in (11) must be accounted for. In the proof of Nielsen (2003, Theorem 9.2) the expression (16) is used with $\varepsilon_{t}$ replaced by a random walk process:

$$
\begin{array}{r}
\frac{1}{T} \beta^{T-2} \sum_{t=1}^{T} \Delta X_{t-1}\left(\sum_{s=0}^{t-1} \varepsilon_{s}\right)=\frac{1}{T} z_{T} \sum_{t=0}^{T-1} \beta^{t}\left(\sum_{s=0}^{T-(t+1)} \varepsilon_{s}\right) \\
+\frac{1}{T} \sum_{t=0}^{T-1}\left[\beta^{t}\left(z_{T-t}-z_{T}\right)\left(\sum_{s=0}^{T-(t+1)} \varepsilon_{s}\right)\right],
\end{array}
$$

and it follows from that proof that this expession is asymptotically vanishing, with the second term on the right hand side of (17) vanishing at an exponential rate.

The numerically unstable terms in each of the $S_{i j}$ expressions can now be approximated by well-behaved quantities derived from Anderson (1959), and so simulation of the $S_{i j}$ terms is possible by using the right hand side expressions of (15), (16) and (17) as opposed to the left hand side quantity. This allows simulation of the likelihood ratio test statistic, and the accuracy of this approximation in small samples is analysed in the next Section using Monte Carlo methods. 
Table 2: Expected value of likelihood ratio test statistic for range of explosive and non-explosive parameterisations, simulated using the idea of Section 3 for $\Gamma \notin[0,2]$. Based on $10^{6}$ replications

\begin{tabular}{|c|c|c|c|c|c|c|c|}
\hline \multirow[b]{2}{*}{$\Gamma$} & \multicolumn{7}{|c|}{$\mathrm{T}$} \\
\hline & 6 & 12 & 24 & 48 & 96 & 192 & $\infty$ \\
\hline$-8 / 3$ & 1.638 & 1.358 & 1.244 & 1.189 & 1.164 & 1.152 & 1.142 \\
\hline$-4 / 3$ & 1.829 & 1.459 & 1.297 & 1.216 & 1.177 & 1.159 & $\vdots$ \\
\hline$-2 / 3$ & 1.686 & 1.671 & 1.402 & 1.271 & 1.205 & 1.172 & $\vdots$ \\
\hline$-1 / 3$ & 1.656 & 1.592 & 1.615 & 1.378 & 1.259 & 1.199 & $\vdots$ \\
\hline$-1 / 6$ & 1.689 & 1.445 & 1.618 & 1.594 & 1.368 & 1.254 & $\vdots$ \\
\hline$-1 / 12$ & 1.707 & 1.463 & 1.400 & 1.645 & 1.584 & 1.363 & $\vdots$ \\
\hline$-1 / 24$ & 1.715 & 1.479 & 1.411 & 1.382 & 1.662 & 1.582 & 1.142 \\
\hline 0 & 1.722 & 1.491 & 1.437 & 1.412 & 1.405 & 1.404 & 1.402 \\
\hline $1 / 24$ & 1.727 & 1.495 & 1.435 & 1.385 & 1.321 & 1.255 & 1.142 \\
\hline $1 / 12$ & 1.730 & 1.490 & 1.409 & 1.327 & 1.255 & 1.207 & $\vdots$ \\
\hline $1 / 6$ & 1.730 & 1.464 & 1.344 & 1.257 & 1.205 & 1.177 & $\vdots$ \\
\hline $1 / 3$ & 1.706 & 1.390 & 1.267 & 1.207 & 1.175 & 1.160 & $\vdots$ \\
\hline $2 / 3$ & 1.607 & 1.293 & 1.212 & 1.175 & 1.158 & 1.150 & $\vdots$ \\
\hline $4 / 3$ & 1.439 & 1.226 & 1.179 & 1.158 & 1.148 & 1.145 & $\vdots$ \\
\hline $8 / 3$ & 1.269 & 1.150 & 1.136 & 1.135 & 1.136 & 1.138 & 1.142 \\
\hline
\end{tabular}

\section{Simulation Results}

Table 2 gives a revised version of Table 1 where the simulations of the explosive cases, $|1-\Gamma|>1$, have been done using the method outlined in Section 3. The numerical instability found in Table 1 does not show up, and it is found that the figures with normal font are exactly the same since the same random numbers have been used in the two Tables.

\subsection{Simulations of Asymptotically Vanishing Terms}

Some attention to the four vanishing terms of equations (15), (16) and (17) is required. They vanish for large $T$, and indeed it was found that for $\gamma=\Gamma T<-16$ each of these terms has simulated expectation and standard deviation of less than $10^{-3}$. Hence convergence of the expressions in the equations (15), (16) and (17) is achieved very quickly. 
Table 3: Summary statistics of the local asymptotic distribution. Expectation and variance are computed from finite sample distribution expectation and variance by fitting polynomials in $T^{-1}$, and the quantiles are generated by fitting a Gamma distribution. The $\gamma=0$ and $\gamma=\infty$ values are drawn from the fixed asymptotic approach, the rest from the local asymptotic method.

\begin{tabular}{lccccccccccc}
\hline$\gamma=\Gamma T$ & 0 & $-1 / 2$ & -1 & -2 & -4 & -8 & -16 & -32 & -64 & -128 & $\infty$ \\
\hline Expectation & 1.402 & 1.373 & 1.360 & 1.365 & 1.670 & 1.574 & 1.357 & 1.248 & 1.193 & 1.168 & 1.142 \\
Variance & 3.097 & 3.009 & 3.014 & 3.036 & 3.718 & 3.792 & 3.043 & 2.637 & 2.424 & 2.322 & 2.221 \\
Shape & 0.634 & 0.627 & 0.614 & 0.614 & 0.750 & 0.653 & 0.605 & 0.591 & 0.587 & 0.588 & 0.587 \\
$90 \%$ Quantile & 3.60 & 3.536 & 3.519 & 3.532 & 4.124 & 4.015 & 3.523 & 3.257 & 3.118 & 3.052 & 2.98 \\
$95 \%$ Quantile & 4.94 & 4.864 & 4.854 & 4.872 & 5.544 & 5.493 & 4.868 & 4.516 & 4.326 & 4.235 & 4.13 \\
\hline
\end{tabular}

In the region $-16 \leq \gamma<0$ these vanishing asymptotic terms can be quite considerable in size. Investigating these terms for a slightly larger array of parameter values and sample sizes than reported, the extreme cases were as follows: for equation (15) the extreme mean and standard deviation are -221.055 and 370.216 respectively; in equation (16) mean -17.841 and standard deviation 10.078 and in equation (17) mean 0.115 and standard deviation 186.949. The reason for the deteriorating quality of the approximation is that when $\gamma$ is close to zero and $\beta=1 /(1-\Gamma)$ close to unity, the explosive elements are no longer dampened. Consideration of Table 1 though shows that this is not a numerical problem: in the region $-16 \leq \gamma \leq 0$ there are no numerical instabilities in computing the expressions in (15), (16) and (17) when using double precision.

\subsection{Evaluating the Asymptotic Result}

Table 2 indicates that the asymptotic result of Theorem 2.1 of Section 2.3 holds. This is a fixed parameter result and indeed when reading the Table row-wise for any $\Gamma \neq 0$ convergence towards the asymptotic value of 1.142 is seen. This limiting value was calculated analytically by Nielsen (1997). It is also seen that the convergence is slower the closer $\Gamma$ is to zero and hence to the non-continuity in the asymptotic distribution.

\subsection{Approximating the Finite Sample Distribution}

In the analysis of the difference stationary region, $|1-\Gamma|<1$, by Nielsen (2004) the local asymptotic approximation of Theorem 2.2 was found to work rather well. This is found also be the case in the explosive region, as seen by reading Table 2 diagonally and thus keeping $\gamma=\Gamma T$ fixed. That is, when $\Gamma<0$ the diagonals from the lower left towards upper right, whereas for $\Gamma>0$ the diagonals from upper left to lower right are followed. For finite $T$, there is much less variation reading along these diagonals than along the rows, implying the local asymptotic distribution is a 
Figure 1: Expected value of fixed and local asymptotic approximating distributions with finite distribution for various parameterisations, with $T=24$ and so $\Gamma=\gamma / 24$. Finite distribution based on $10^{6}$ repetitions.

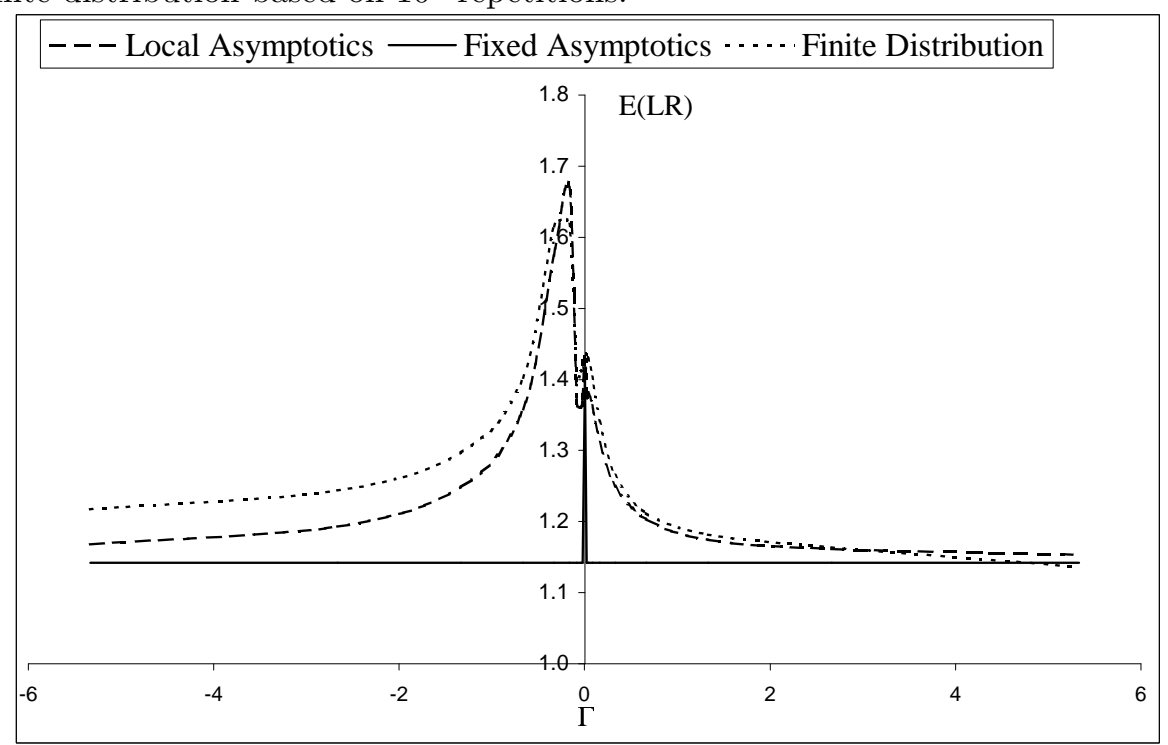

more suitable approximation.

Table 3 reports summary statistics for the local asymptotic distribution when $\Gamma \leq 0$. For $\Gamma<0$ the expectation and variance figures are found by a response surface analysis of the numbers along the diagonals in extended versions of Table 2 for both simulated means and variances. Following Nielsen $(1997 ; 2004)$ the $90 \%$ and $95 \%$ quantiles are then found by fitting Gamma distributions by matching the first two moments. The shape parameters of these Gamma distributions are also reported. The entries for $\Gamma=0$ and $\Gamma=\infty$ are taken from Nielsen $(1997 ; 2004$, Table 2).

Figure 1 plots the expected values of the finite, fixed asymptotic and local asymptotic distributions for a range of $\Gamma$ values when $T=24$. In this situation the statistical model has three parameters, so there are eight observations per parameter, corresponding to many empirical studies. The fixed parameter asymptotic distribution has a discrete jump at $\Gamma=0$, whereas the local asymptotic distribution and the finite sample distribution appear to be continuous over the entire $\Gamma$ space, albeit with two local maxima. The departure of the local asymptotic and finite sample distributions from the fixed asymptotic distribution can again be seen, and is most marked just below zero, which corresponds to the $(\Gamma, T)$ pair of $(-1 / 6,24)$ hence $\gamma=-4$. As the local asymptotic distribution differs from the fixed distribution in a similar fashion to the finite sample distribution, this again suggests it provides a much better approximation to the finite order distribution.

Table 4 reports rejection frequencies for tests based on the $95^{\text {th }}$ quantile of the 
Table 4: Probabilities $\mathrm{P}\left(\mathrm{LR}>c_{\infty}\right)$ for the two approximating asymptotic distributions, along with Bartlett Corrected probabilities, and Bartlett Factors. The finite distribution is taken to be $T=24$. Based on $10^{6}$ repetitions.

\begin{tabular}{lrrrrrrrrr}
\hline$\Gamma$ & $-1 / 48$ & $-1 / 24$ & $-1 / 12$ & $-1 / 6$ & $-1 / 3$ & $-2 / 3$ & $-4 / 3$ & $-8 / 3$ & $-16 / 3$ \\
$\gamma=\Gamma T$ & $-\frac{1}{2}$ & -1 & -2 & -4 & -8 & -16 & -32 & -64 & -128 \\
$\begin{array}{l}\text { Fixed Asymptotics } \\
\text { Prob - asymp }\end{array}$ & 0.079 & 0.078 & 0.077 & 0.094 & 0.098 & 0.077 & 0.066 & 0.061 & 0.058 \\
Prob - Bartlett & 0.048 & 0.048 & 0.048 & 0.040 & 0.044 & 0.048 & 0.049 & 0.050 & 0.050 \\
Bartlett Factor & 1.249 & 1.235 & 1.226 & 1.417 & 1.414 & 1.228 & 1.136 & 1.089 & 1.066 \\
Local Asymptotics & & & & & & & & & \\
Prob - asymp & 0.055 & 0.055 & 0.053 & 0.047 & 0.052 & 0.053 & 0.054 & 0.055 & 0.548 \\
Prob - Bartlett & 0.050 & 0.050 & 0.050 & 0.051 & 0.049 & 0.049 & 0.049 & 0.050 & 0.050 \\
Bartlett Factor & 1.039 & 1.038 & 1.026 & 0.969 & 1.026 & 1.033 & 1.039 & 1.043 & 1.042 \\
\hline
\end{tabular}

fixed and local approximating asymptotic distributions. The first row of Table 4 shows strong size distortions for the non-explosive region under fixed asymptotics, as the rejection probabilities are all well over $5 \%$, with the most extreme rejection probability of nearly $10 \%$ when $\gamma$ is about -4 .

Table 4 also reports Bartlett corrections of the two asymptotic distributions. Following Bartlett (1937) the idea is to scale the approximating asymptotic distribution in order that it better matches the small sample distributions. If the quantiles of the finite and asymptotic distributions are approximately proportional then the approximation can be improved by scaling it by the ratio of finite and asymptotic expectations of the test statistic. Bartlett showed that the scaling factor used should be the ratio of finite distribution expected values to asymptotic distribution expected value. Lawley (1956) has shown that in testing situations with $n$ independent observations, Bartlett corrections eliminate terms in $n^{-1}$ from asymptotic expansions of the moments of the likelihood ratio test statistic for large $n$. For the unit root test, however, observations are not even stationary, and so the conditions for Lawley's (1956) result do not hold, but Nielsen (1997) has proved that in the first order autoregressive model a Bartlett correction reduces the absolute value of the second order terms for at least the first four moments.

From the second row of Table 4 for fixed parameter asymptotics, the rejection frequencies are improved dramatically by Bartlett correction, with most probabilities lying in the region $4.8-5 \%$. There is a slight over-correction in that all the values are equal to or below $5 \%$, with a probability as low as $4 \%$ for $\Gamma=-1 / 6$. The row below this reports the relevant Bartlett factors, which vary considerably over the range of parameter values considered in line with the variation of the slope parameters in Table 3 .

Turning to consider local asymptotics, there is an improvement over the fixed parameter asymptotic distribution even before correcting: all rejection probabilities 
are within half a percentage point of $5 \%$, although all bar one are above $5 \%$. The one value below $5 \%$ is for $\Gamma=-1 / 6$, and this is where there is the previously noted spike in the size and expected values of the finite sample and local asymptotic distributions. Table 4 shows the Bartlett corrections, where all the corrected probabilities are within a tenth of a percentage point of $5 \%$ and over half of them are even within a twentieth of a percentage point. The associated Bartlett factor is reported in the row below the corrected probability; all are close to unity but once again with change from above 1 to below 1 around $\gamma=-4$. While this Bartlett factor appears to be uniformly bounded it shows fluctuations with a peak of $\gamma$ close to -4 .

Hence from the results of Table 4, size distortions can be all but eradicated for the likelihood ratio test when the process has an explosive root by using local asymptotics and Bartlett corrections. These results closely mimic those of Nielsen (2004) for the non-explosive region.

\section{Conclusions}

In this paper, the properties of unit root tests in the explosive second order autoregressive model have been analysed by simulation. Standard simulation techniques lead to numerical instability in this situation. The poor numerical accuracy of this procedure has been overcome using the approximations described in Anderson (1959), which were shown to be good approximations even in small samples. This lead to accurate simulations of the distribution of the likelihood ratio test for the explosive region, supporting the mathematical proofs of Nielsen (2001) relating to the asymptotic distributional approximations in the explosive region. It was also shown that for the explosive case the finite sample properties of this fixed parameter approximation can be improved by a local asymptotic approximation along the lines of Nielsen (2004), who considered the difference stationary case.

\section{Acknowledgements}

The simulation results were generated using Ox (see Doornik, 1999).

\section{References}

Anderson, T.W. (1959), 'On asymptotic distributions of estimates of parameters of stochastic difference equations', The Annals of Mathematical Statistics 30, 676687.

Bartlett, M.S. (1937), 'Properties of sufficiency and statistical tests', Proceedings of the Royal Society of London, Series A 160, 268-282.

Basawa, I.V., A.K. Mallik, W.P. McCormick and R.L. Taylor (1989), 'Bootstrapping explosive autoregressive processes', The Annals of Statistics 17, 1479-1486. 
Doornik, J.A. (1999), Object-Orientated Matrix Programming Using Ox 2.1, Timberlake, London.

Lai, T.L. and C.Z. Wei (1983), 'A note on the martingale sequences satisfying the local Marcinkeiwicz-Zygmund condition', Bulletin of the Institute of Mathematics, Academia Sinica 11, 1-13.

Lawley, D.N. (1956), 'A general method for approximation to the distribution of the likelihood ratio criteria', Biometrika 43, 295-303.

Nielsen, B. (1997), 'Bartlett correction of the unit root test in autoregressive models', Biometrika 84, 500-504.

Nielsen, B. (2001), 'The asymptotic distribution of unit root tests of unstable autoregressive processes', Econometrica 69, 211-219.

Nielsen, B. (2003), Strong consistency results for least squares estimators in general vector autoregressions with deterministic terms. Mimeo, Nuffield College, Oxford. To appear in Econometric Theory.

Nielsen, B. (2004), 'On the distribution of likelihood ratio test statistics for cointegration rank', Econometrics Reviews 23, 1-23. 